Relations industrielles

Industrial Relations

\title{
Décisions rendues par le CRTC
}

\section{Luc Martineau}

Volume 34, numéro 4, 1979

URI : https://id.erudit.org/iderudit/029018ar

DOI : https://doi.org/10.7202/029018ar

Aller au sommaire du numéro

Éditeur(s)

Département des relations industrielles de l'Université Laval

ISSN

0034-379X (imprimé)

1703-8138 (numérique)

Découvrir la revue

Citer cet article

Martineau, L. (1979). Décisions rendues par le CRTC. Relations industrielles /

Industrial Relations, 34(4), 813-823. https://doi.org/10.7202/029018ar

Tous droits réservés @ C Département des relations industrielles de l'Universite Laval, 1979
Ce document est protégé par la loi sur le droit d'auteur. L'utilisation des services d'Érudit (y compris la reproduction) est assujettie à sa politique d'utilisation que vous pouvez consulter en ligne.

https://apropos.erudit.org/fr/usagers/politique-dutilisation/ 
intégrer dans le corps de l'article 20 qui consacre le droit à l'accréditation, l'équivalent de l'article 134(1) du Code canadien qui stipule

«134. (1) Nonobstant toute autre disposition de la présente Partie, lorsque le Conseil est convaincu qu'un syndicat est dominé ou influencé par un employeur au point que son aptitude à représenter les employés de l'employeur aux fins des négociations collectives est diminuée,

a) il ne doit pas accréditer le syndicat à titre d'agent négociateur d'une unité groupant des employés de cet employeur; et

b) toute convention collective conclue par le syndicat et l'employeur et qui s'applique à tout ou partie de ces employés est censée, aux fins de la présente Partie, ne pas être une convention collective.»

\section{Décisions rendues par le Conseil canadien des relations du travail}

\section{Employés ajoutés à une unité de négociation existante}

Le Conseil a accueilli une requête en révision présentée par le Syndicat canadien des télécommunications transmarines (SCTT) en vertu de l'article 119 du Code qui demandait d'ajouter à l'unité de négociation existante un groupe considérable d'employés exerçant des fonctions comparables à celles du premier groupe mais dont les qualifications techniques étaient plus exigeantes.

Constatant que la requête ne modifiait pas la nature, l'essence et la portée intentionnelle de l'unité de négociation existante et d'autre part, que le syndicat requérant a pu démontrer qu'il était désiré par une majorité globale des employés dans l'unité, le Conseil a décidé d'ajouter les employés visés par ladite requête, sauf une restriction à l'égard des professionnels, sans tenir compte de leur désir.

Syndicat canadien des télécommunications transmarines et Téléglobe Canada et al.

Dossiers 530-179, 530-180 et 555-703; décision du 17 juillet 1979 (no 198); panel du Conseil: Me Marc Lapointe, président, MM. Jacques Archambault et Norman Bernstein, membres.

* Cette chronique a été préparée par Luc Martineau, avocat, adjoint légal au président du Conseil canadien des relations du travail. 


\section{FAITS SAILLANTS}

L'entreprise impliquée est une société de la Couronne oeuvrant dans le champ des télécommunications. Entre 1952 et 1975, cette dernière prit une expansion très considérable et ce, surtout à compter de 1973: de 245 employés qu'elle engageait alors en 1952, ce nombre était passé à 1177 le 29 juin 1976, date à laquelle le SCTT faisait parvenir au Conseil sa requête en révision.

Dans l'espèce, ladite requête visait à modifier le contenu d'une unité de négociation ayant fait l'objet d'une ordonnance d'accréditation par le Conseil le 26 janvier 1976. Celle-ci avait été émise sur l'insistance des parties en cause pressées à cette époque de régler les différends qui les opposaient suite aux négociations entourant le renouvellement de leur convention collective. Le Conseil avait alors accepté de sanctionner l'entente des parties bien que la définition de l'unité de négociation proposée ait été énumérative, sous réserve de réviser par la suite sa décision en vertu de l'article 119 du Code.

Avant cette ordonnance d'accréditation de 1976, à peu près cette unité de négociation était représentée par l'Overseas Communications Union local 272, affiliée au Congrès du travail du Canada qui détenait un certificat d'accréditation depuis le 8 août 1952, certificat qui n'avait jamais été modifié. En 1975, Overseas Communications Union local 272, se transformait en association indépendante de toute affiliation à une centrale syndicale, et devenait le Syndicat canadien des télécommunications transmarines (SCTT). Ce dernier déposait le 3 septembre 1975 une requête en accréditation qui fut agréée comme mentionné plus haut, par le Conseil le 26 janvier 1976.

L'enquête menée par le Conseil dans ce dossier devait révéler qu'en avril 1977, il y avait environ 350 employés dans l'unité existante (par rapport à 154 en 1952) et que les employés assignés aux classifications dont le syndicat recherchait l'inclusion par sa requête en révision totalisaient 176 personnes pour un grand total de 526 employés.

\section{QUESTIONS SOULEVÉES}

Cette décision particulièrement importante met en jeu la compétence du Conseil de modifier de son propre chef, et sérieusement, l'ampleur numérique d'une unité de négociation. Elle soulève aussi la question de l'expression du désir des employés visés par la requête du syndicat et qui doivent être ajoutés à l'unité existante. Ont-ils le droit d'être consultés et comment? Jusqu'où l'impact de restructurations majeures par un employeur peut-il influer sur le devoir du Conseil de décréter, de maintenir ou de modifier les unités de négociation?

\section{PRINCIPAUX MOTIFS DE DÉCISION}

\section{L'interprétation de l'article 119}

C'est en vertu de l'article 119 du Code que le syndicat requérant s'est appuyé pour demander au Conseil d'ajouter à l'unité de négociation actuelle diverses nouvelles classifications afin d'en faire une unité plus appropriée. 
Cet article se lit comme suit:

«le Conseil peut réviser, annuler ou modifier toute décision ou ordonnance rendue par lui et peut entendre à nouveau toute demande avant de rendre une ordonnance relative à cette dernière.»

Le Conseil a jugé que le libellé de cette disposition lui conférait juridiction pour entendre la requête du syndicat, a rappelé que cette demande entrait dans le cadre des situations où l'exercice des pouvoirs du Conseil en vertu de l'article 119 était susceptible d'être invoqué, celles-ci pouvant être de trois types principaux:

(1)- l'agent négociateur peut désirer que son certificat d'accréditation soit révisé de manière à ce que l'unité de négociation actuelle soit agrandie ou substantiellement modifiée, ce qui avait pour résultat d'affecter la nature et la portée intentionnelle de ce certificat;

(2)- suite à un concours de circonstances survenues postérieurement à la décision ou à l'ordonnance du Conseil, certains éclaircissements ou certaines modifications peuvent se faire sentir sans pour autant que la nature et la portée intentionnelle de cette décision ou de cette ordonnance ne s'en trouvent affectées;

(3)- une partie demande simplement au Conseil de reconsidérer sa décision parce qu'elle serait erronée.

L'initiative de demander au Conseil de réviser l'une de ses décisions pour les motifs ci-haut mentionnés revient normalement aux parties, mais rien n'empêche le Conseil d'agir proprio motu et de prendre lui-même cette initiative puisqu'il est clair que lorsque le législateur ne le stipule pas et c'est le cas pour l'article 119, le Conseil peut agir de son propre chef, sans demande ou requête de parties intéressées.

\section{Le désir des employés}

Dans le premier type de situations la nature et la portée intentionnelle du certificat d'accréditation sont affectées. Dès lors, la demande de révision formulée au Conseil en vertu de l'article 119 est fondamentalement du même genre que celui d'une requête en accréditation. Pour cette raison, le Conseil considère que dans ces cas il devra tenir compte du désir des employés ajoutés et de ceux appartenant à l'unité existante. Par contre dans le deuxième type mentionné qui ne modifie pas radicalement l'effet de la décision du Conseil, seule l'obtention d'une majorité globale dans l'unité de négociation «agrandie» devrait suffire. Faisant la lumière sur les critères qui seront utilisés pour déterminer si la requête en révision modifie la nature et l'effet de l'ordonnance originale, le Conseil s'est référé au concept de la portée intentionnelle du certificat original d'accréditation qui depuis un certain nombre d'années a été développé dans la province de Québec.

\section{L'époque propice pour procéder par demande en révision et non par accréditation}

Qu'il s'agisse du premier type de situations ou du deuxième, que la portée intentionnelle du certificat d'accréditation soit affectée ou non, une requête en révision pourra être faite en tout temps. Le Conseil désire ainsi éviter que les nouveaux employés de l'entreprise soient toujours acculés à la seule option de se regrouper dans une unité séparée en les obligeant à respecter les délais qui sont prévus au Code pour la présentation des requêtes en accréditation, ce qui d'autre part pourrait résulter en une prolifération automatique des unités de négociation. Cependant, le 
Conseil ne modifiera pas la date d'expiration de la convention collective originale sauf si les parties le demandent conjointement.

\section{Conclusion:}

Le Conseil en étant venu à la conclusion que la requête du syndicat ne changeait pas radicalement la nature du certificat d'accréditation original et compte tenu du caractère représentatif global du requérant parmi les membres de l'unité de négociation modifiée, il a décidé de faire droit à la demande en révision. Les employés visés par ladite requête, sauf une restriction à l'égard de certains professionnels, seront ajoutés à l'unité de négociation existante.

\section{Création de nouvelles classifications dans l'entreprise}

Le Conseil a accueilli une requête en révision présentée par le Syndicat des travailleurs en télécommunications en vertu de l'article 119 du Code demandant l'amendement d'un certificat d'accréditation émis le 23 février 1949 en faveur du prédécesseur du requérant, Federation of Telephone Workers of British Columbia.

S'en référant cependant à la portée intentionnelle du certificat d'accréditation de 1949, le Conseil a décidé que la présente ordonnance modifiée ne devait couvrir que l'unité de négociation actuellement visée par la convention collective. L'unité de négociation est décrite dans un langage universel, à la suite de laquelle suit une série d'exclusions qui reflètent les classifications ne tombant pas dans le champ d'application de la convention collective.

En vertu des pouvoirs qui lui sont accordés en vertu de l'article 121 du Code, le Conseil a aussi inclus dans ladite ordonnance une procédure pour le règlement des différends qui pourront surgir entre les parties à l'occasion de la création de postes tombant en dehors de la portée intentionnelle de la nouvelle unité de négociation.

Quant à la portée intentionnelle de ce nouveau certificat, le Conseil l'interprète comme devant couvrir tous les nouveaux postes qui pourront être créés au sein de l'entreprise dans l'avenir. L'employeur aura le fardeau de prouver au Conseil que lesdits postes ne devraient pas faire partie de l'unité de négociation.

Saisi d'un renvoi formulé en vertu de l'article 158 du Code, le Conseil s'est aussi prononcé sur l'applicabilité de la convention collective quant aux fonctions exercées par certaines personnes et ayant fait l'objet de mésententes entre les parties.

Syndicat des travailleurs en télécommunications et British Columbia Telephone Company et al.

Dossiers 530-363, 530-371, 530-432 et 610-15, décision du 22 août 1979 (no 206);

panel du Conseil: Me James E. Dorsey, vice-président, Me Robert J. Arseneau et M. Hugh R. Jamieson, membres.

\section{FAITS SAILLANTS}

L'entreprise opérée par l'employeur en est une qui a été déclarée être à l'avantage général du Canada par le Parlement canadien en vertu de l'article 9.2(10) (c) de l'Acte de l'Amérique du Nord Britannique, ce qui explique pourquoi les relations de travail de ladite entreprise tombent dans le champ d'application du Code Canadien du Travail. En effet, les relations de travail de toutes les autres entreprises de 
téléphone du Canada, à l'exception de cette dernière et de Bell Canada, sont régies par des lois provinciales.

Le 23 février 1949, l'ancien Conseil fédéral rendait une ordonnance d'accréditation déterminant le contenu approprié de l'unité de négociation en des termes généraux, à la suite de laquelle suivait une lọngue liste d'exclusions qui apparemment ne couvraient que 233 personnes.

En 1964, le syndicat présentait au Conseil une demande en accréditation pour représenter un groupe d'ingénieurs assistants qui ne fit jamais l'objet d'une ordonnance du Conseil, le syndicat ayant décidé de se désister de sa requête parce qu'il avait par la suite conclu avec l'employeur une convention collective couvrant ce groupe d'employés.

En 1967, le syndicat demanda au Conseil de se prononcer sur l'inclusion de certaines nouvelles classifications créées depuis 1949 dans l'unité de négociation, ce qu'il fit mais sans se prononcer sur la question du désir des employés. Cependant, plus tard en 1970, suite à une requête en révision portée en vertu de l'article 61(2) de l'ancienne loi, le Conseil d'alors exprimait clairement sa politique de traiter la demande du syndicat comme s'il s'agissait d'une requête en accréditation et qu'en l'absence d'entente entre les parties sur l'inclusion de ces personnes dans l'unité de négociation, le syndicat devait démontrer que ces derniers désiraient être représentés par celui-ci. Le syndicat se désista alors de ses demandes et chercha d'autres moyens pour résoudre ses difficultés.

C'est ainsi qu'il eu recours à un tribunal d'arbitrage qui décida que ces personnes faisaient partie de l'unité de négociation et dont la décision fut confirmée par la Cour d'Appel de la Colombie Britannique. La procédure imposée par le tribunal d'arbitrage pour le règlement des différends fut suivie par l'employeur qui pendant un certain temps informait le syndicat de tout changement dans les classifications, jusqu'à ce que le nouveau Conseil reçoive de d'autres syndicats des demandes en accréditation pour représenter des superviseurs au sein de l'entreprise.

Le syndicat demande alors d'intervenir dans ces dossiers pour s'y objecter au chef que plusieurs des classifications visées appartenaient à l'aire de son propre certificat d'accréditation tel qu'il l'alléguait et le plaidait dans la requête en révision qu'il avait faite en vertu de l'article 119, alors pendante devant le Conseil. La demande du syndicat fut rejetée [voir: (1977) 22 di 507]. Le Conseil jugeait que le syndicat avait pendant de nombreuses années volontairement abandonné son droit de négocier à l'égard de certains employés qui à l'origine pouvaient faire partie de l'unité de négociation. Dans les circonstances, pour étendre l'unité existante afin de couvrir ces employés, le syndicat devait démontrer qu'il avait l'appui majoritaire de ceux-ci.

Quant à la requête en révision comme telle, elle était par la suite rejetée par le Conseil le 11 mai 1978 [voir: (1978) 2 Can LRBR 387].

Suite à cette décision, le syndicat a fait une nouvelle demande sous l'article 119 au sujet de la portée de l'unité de négociation. Le syndicat ayant aussi décidé de porter ces problèmes en arbitrage, l'employeur a répliqué en faisant une demande de renvoi au Conseil sous l'article 158 du Code prétendant qu'il s'agissait d'une matière non-arbitrable. C'est au sujet de ces demandes que le Conseil s'est prononcé dans la présente décision et dont le sommaire apparaît plus haut. 


\section{QUESTIONS SOULEVÉES}

Quelle était la portée intentionnelle du certificat d'accréditation de 1949? Est-ce que le Conseil devait faire droit à la requête en élargissement de l'unité de négociation présentée par le syndicat compte tenu des circonstances particulières de cette affaire? Comment les parties en viendraient-elles à régler les mésententes continuelles surgissant à l'occasion de la création de nouvelles classifications au sein de l'entreprise? Le Conseil avait-il juridiction pour insérer dans son ordonnance les termes d'une procédure de règlement de tels différends entre les parties?

\section{PRINCIPAUX MOTIFS DE DÉCISION}

La portée intentionnelle du certificat d'accréditation original

Nonobstant le fait que l'ordonnance d'accréditation émise par le prédécesseur du présent Conseil en 1949 ait voulu décrire l'unité de négociation en des termes généraux afin de couvrir tous les employés de l'employeur à l'exclusion du personnel de direction, des professionnels et de ceux exerçant des fonctions confidentielles ou de surveillance, ce qui était assez exceptionnel pour l'époque, il appert cependant qu'il faille interpréter ce langage à la lumière de ce qui était compris entre les parties.

Le comportement des parties démontre bien qu'ils n'ont jamais interprété cette définition de l'unité de négociation décrite au certificat de la manière large dont il serait perçu aujourd'hui si la même ordonnance était de nouveau émise par le présent Conseil. En fait si l'on jette un regard sur les exclusions mentionnées dans ce certificat, on se rend compte que la portée intentionnelle dudit certificat était seulement de couvrir les employés faisant partie de l'unité de négociation sur laquelle les parties avaient réussi à s'entendre à cette époque. L'ordonnance du Conseil ne faisait que sanctionner l'entente intervenue entre les parties. D'ailleurs, il n'a jamais fait d'enquête pour déterminer si les exclusions insérées dans ladite ordonnance relevaient de l'exercice par ces personnes de fonctions de direction, de surveillance, ou encore à caractères professionnel ou confidentiel.

Cette perception qu'ont pu avoir les parties face à la portée réelle de ce certificat est vérifiable par leurs agissements postérieurs, tels par exemple, le fait que pendant de nombreuses années les droits de négociation du syndicat face à la représentation de certains groupes d'employés se soient faits sentir par un mécanisme de reconnaissance volontaire de ceux-ci par l'employeur, plutôt que de revenir devant l'ancien Conseil pour faire interpréter ledit certificat pour couvrir ces employés, bien que le présent Conseil doive admette que dans ces cas le syndicat ait pu se sentir désavantagé à cause de la politique restrictive suivie dans le passé au sujet des demandes en révision.

\section{L'effet de la nouvelle ordonnance émise par le Conseil}

Ayant considéré la portée intentionnelle du certificat original, le Conseil a refusé d'agrandir l'unité de négociation actuelle.

L'actualité de cette unité de négociation trouve son reflet dans la reconnaissance volontaire au sein de l'entreprise de certaines nouvelles classifications qui ont été créées depuis 1949 et pour lesquelles le syndicat requérant a acquis des droits de 
représentation. Cependant, le Conseil n'est pas prêt, en l'absence d'une preuve démontrant que le syndicat détient le caractère majoritaire requis au sein du groupe d'employés qui n'est pas visé par le certificat original ou autrement reconnu par l'employeur comme faisant partie de l'unité de négociation existante, à les inclure dans la nouvelle ordonnance d'accréditation.

Ainsi donc l'ordonnance qui a été émise dans le présent cas couvrira «tous les employés» de l'entreprise à l'exclusion de ceux qui exercent des fonctions dont le contenu n'est pas actuellement couvert par une convention collective. En agissant de la sorte, le Conseil espère rétablir un équilibre qui semble avoir été perdu au cours des années concernant le champ d'application de l'unité de négociation.

Concernant la portée intentionnelle de cette nouvelle unité de négociation, appropriée dans les circonstances, le Conseil a précisé dans ses motifs de décision, qu'il la voyait au point de départ comme devant couvrir toutes les nouvelles classifications pouvant par la suite être créées. Ce sera à l'employeur d'établir qu'elles ne devraient pas être incluses et de repousser la présomption pesant contre lui. En déterminant si telle fonction est visée par l'ordonnance actuelle, lorsque requis, le Conseil étudiera sa similitude avec les fonctions ou classifications qui ont été incluses dans le certificat et non avec celles qui ont été exclues, bien qu'en surface cette approche puisse donner lieu à certaines contradictions.

L'insertion d'une procédure de règlement des différends pouvant naître entre les parties à l'occasion de la création de nouvelles classifications

Les faits saillants rapportés précédemment font bien apparaître les difficultés qu'ont dues encourir les parties à chaque fois qu'une nouvelle classification était créée par l'employeur.

Afin de résoudre ces difficultés, le Conseil a décidé d'inclure à la suite de son ordonnance une procédure de règlement des différends que les parties devront suivre, en l'absence d'entente au contraire dans une convention collective, lorsqu'il surviendra des mésententes lors de la création de nouvelles classifications.

Les termes de cette procédure imposée à l'avenir aux parties prévoient entre autres qu'à chaque fois que l'employeur désire créer une nouvelle classification ou modifier le titre donné à l'une des classifications tombant en dehors du champ d'application du certificat d'accréditation, il devra le notifier par écrit à l'agent négociateur. Celui-ci aura quatorze jours pour indiquer à l'employeur qu'il entend contester le changement, à défaut de quoi il sera présumé avoir accepté ledit changement. Dans les délais indiqués par le Conseil, les parties devront se rencontrer et si à l'intérieur de tels délais, elles ne se sont pas entendues, le différend pourra être porté devant le Conseil qui décidera de l'inclusion ou de l'exclusion du poste contesté.

Le Conseil a invoqué l'article 121 du Code pour donner un effet légal et compulsif à l'inclusion de cette procédure dans une ordonnance rendue par celui-ci. Cet article se lit comme suit:

«Le Conseil exerce les pouvoirs et fonctions que lui attribue la présente Partie ou qui peuvent être nécessaires à la réalisation des objets de la présente Partie, et notamment, sans restreindre la portée générale de ce qui précède, il rend des ordonnances comportant obligation de se conformer aux dispositions de la présente Partie, de tout règlement pris sous son régime ou de toute décision rendue dans une affaire dont il est saisi.» 
Mentionnons finalement que le Conseil laisse libre cours aux parties de s'entendre entre elles sur une autre procédure alternative et qu'advenant tout conflit entre ces deux procédures, le problème pourra toujours être référé au Conseil en vertu de l'article 158 du Code si les parties ont choisi de porter leurs mésententes devant un arbitre ou un tribunal d'artibrage privé.

\section{Congédiement illégal et mitigation des dommages}

Suite à une ordonnance de réintégration et de compensation dans le cas de deux congédiements illégaux, la compagnie-employeur, Victoria Flying Services, a été vendue à trois compagnies différentes. Le Conseil a décidé qu'il y avait eu vente au terme de l'article 144 du Code et que les trois compagnies étaient tenues de payer conjointement et solidairement la compensation allouée aux employés congédiés. Le Conseil a également décidé que les employés congédiés illégalement n'étaient pas obligés de mitiger leurs dommages et de justifier qu'ils avaient fait tous les efforts raisonnables pour se trouver un nouvel emploi.

Fraternité canadienne des cheminots, employés des transports et autres ouvriers et Victoria Flying Services Ltd. et al.

Dossiers 585-15, 745-173, 745-174 et 560-21; décision du 29 juin 1979; panel du Conseil: Me Claude H. Foisy, vice-président, Me Robert J. Arseneau et M. Norman Bernstein, membres.

\section{Ordonnance de "cease and desist"}

Le refus concerté de certains employés d'exécuter du temps supplémentaire avant l'expiration des délais prévus au Code réglementant l'exercice du droit de grève constitue des arrêts de travail illégaux.

Le Conseil exerçant la discrétion qui lui est conférée en vertu du Code pour faire émettre des ordonnances de "cease and desist" a déclaré que ces arrêts de travail étaient illégaux et a ordonné aux employés et au syndicat de cesser pour le futur d'en déclarer d'autres ou d'y participer et ce, jusqu'à l'obtention du droit de grève légale.

Le Conseil a mentionné en obiter dictum, qu'après avoir donné l'opportunité à la partie lésée de se faire entendre, il peut ne pas rendre une ordonnance de "cease and desist" même devant des faits démontrant qu'il y a arrêt de travail illégal. Tout dépendra des intérêts supérieurs à satisfaire dans des circonstances données: [EX$T R A I T]$

"Le Parlement canadien cherchait, selon nous, à enrichir l'arsenal de moyens d'action confiés au Conseil pour aider les parties à conclure librement et dans l'ordre des ententes collectives ou à régler dans l'ordre les arrêts de travail durant le cours d'une convention collective. C'est ainsi que ce Conseil interprète la discrétion fondamentale et significative qui lui est laissée dans les articles 182 et 183. Après avoir donné l'opportunité à la partie visée de se faire entendre, le Conseil peut ne pas rendre une ordonnance même devant des faits démontrant qu'il y a arrêt de travail illégal. Tout dépendra des intérêts supérieurs à satisfaire dans des circonstances données: ces intérêts supérieurs peuvent se résumer en mots très simples. Il s'agit de créer ou d'aider à créer une situation de faits la plus susceptible de favoriser des relations ouvrières saines et ordonnées. Pour ce faire, ce Conseil croit qu'il peut se produire des circonstances où un arrêt de travail illégal est la conséquence d'une perturbation dans les relations entre les parties dont il importera d'identifier la cause pour y 
trouver remède. C'est ce qu'il a instruit ses agents de faire dans des rencontres avec les parties avant l'audience publique. On a évoqué ci-haut le succès de cette politique et de la pratique suivie. Mais même en cas d'échec de l'agent du Conseil, il se peut que ce dernier, suite à une audience publique, en arrive à la conclusion qu'il peut agir dans le même sens, soit en assortissant une ordonnance de directives précises tendant à corriger la cause de la perturbation, soit en refusant de faire émaner une ordonnance.» (Décision du Conseil, aux pp. 13-14).

Conseil des Ports Nationaux et Syndicat National des Employés du Port de Montréal et al.

Dossier 725-39; décision du 22 mai 1975 (no 195) panel du Conseil: Me Marc Lapointe, président, MM. Jacques Archambault et Lorne E. Shaffer, membres.

\section{Poursuites pénales pour refus de respecter une ordonnance de "cease and desist"}

Suite à la déclaration du Conseil que certains arrêts de travail dans le Port de Montréal étaient illégaux et que l'ordonnance de "cease and desist" émise précédemment dans le même dossier n'avait pas été respectée, le Conseil a accordé son consentement à l'employeur en vertu de l'article $194 \mathrm{du}$ Code pour que des poursuites pénales soient intentées.

Cependant, le Conseil a limité ce consentement à poursuivre contre le syndicat seulement et il a restreint la période pendant laquelle l'employeur pourra exercer son droit, soit avant la date de la signature de la convention collective renouvellée.

En rendant sa décision, le Conseil a fait part de sa politique générale de refuser dans de tels cas l'autorisation automatique de porter plainte au criminel. Le pouvoir discrétionnaire du Conseil d'autoriser des poursuites pénales en vertu de l'article 194 et/ou encore de déposer son ordonnance à la Cour Fédérale en vertu de l'article 123, ce qui ultimement peut entre autres mener à une condamnation pour mépris de Cour, s'exercera en tenant compte des saines relations de travail: [EXTRAIT].

"Lorsque le Conseil autorise des poursuites sous l'article 194, comme dans le cas où il dépose son ordonnance afin qu'elle puisse être susceptible d'exécution par la Cour fédérale en vertu de l'article 123, il ne sanctionne pas le défaut de se conformer à son ordonnance. Ce n'est pas le Conseil qui rendra le jugement pénal ni la sentence, non plus qu'il ne rendra par exemple une sentence de mépris de Cour.

Comme nous l'avons mentionné plus haut, le pouvoir discrétionnaire du Conseil de se servir des outils mis à sa disposition pour atteindre les fins du Code doit surtout être exercé en tenant compte des buts recherchés par l'ordonnance rendue. En d'autres termes, lorsque le Conseil se demande s'il autorisera des poursuites pénales ou déposera son ordonnance à la Cour fédérale, il doit nécessairement vérifier si l'ordonnance rendue en vertu de l'article 182 ou de l'article 183 avec les directives y ajoutées, a été respectée et si les directives qu'il a fait émaner ont été comprises par une ou par les parties et ont donné les résultats escomptés. C'est la raison pour laquelle, règle générale, nous n'accorderons pas de consentement en vertu de l'article 194, avant de savoir non seulement si l'ordonnance rendue en vertu de l'article 182 a été respectée mais aussi le comportement des parties en termes de saines relations de travail. Si tout est retombé dans l'ordre et que la cause véritable du conflit est solu- 
tionnée, il est peu probable qu'il soit dans l'intérêt de bonnes relations de travail d'autoriser des poursuites pénales." (Décision du Conseil à la page 9).

Conseil des Ports Nationaux et Syndicat National des Employés du Port de Montréal et al.

Dossier 750-2; décision du 22 mai 1979 (no 197); panel du Conseil: Me Marc Lapointe, président, Me Claude H. Foisy, viceprésident et $M$. Lorne E. Shaffer, membre. (Les motifs de décision ont été communiqués par le vice-président).

\section{Maraudage intersyndical et tenue d'un scrutin de représentation}

Le Syndicat des travailleurs en communication du Canada a demandé au Conseil de réviser sa décision à l'effet de lui refuser l'accréditation au motif qu'il aurait commis une erreur de droit.

La requête du syndicat est rejetée parce qu'il n'a pas plaidé ledit point de droit en temps utile.

Cependant, le Conseil a profité de cette occasion pour faire part des remarques supplémentaires suivantes quant à la politique générale du Conseil d'ordonner des scrutins de représentation lorsqu'il y a maraudage intersyndical: [EXTRAIT].

«Pour résumer, le Conseil, avant d'accréditer un syndicat, doit être convaincu que les employés désirent majoritairement être représentés par ce syndicat. Le désir des employés, règle générale, s'évalue à la date du dépôt de la requête en accréditation et ce, par référence à leur adhésion syndicale. Dans le cas d'un syndicat qui tente d'en déplacer un autre la règle générale est à l'effet que nous décréterons un scrutin pour évaluer le désir des employés seulement si le syndicat déplaçant peut prouver qu'au moment du dépôt de sa requête en accréditation la majorité des employés de l'unité accréditée y avait adhéré. Cette façon de procéder tient compte, comme nous l'avons déjà dit, du fait que nous devons assurer la stabilité dans les relations de travail et qu'il n'est pas dans l'intérêt de l'administration du Code de favoriser des maraudages, ni de favoriser une formation au détriment de l'autre. La règle du vote, même en cas de majorité au moment du dépôt, tient compte du tiraillement des employés ballotés entre plusieurs syndicats et soumis à de fortes pressions. Nous avons souvent vu dans de telles circonstances, des syndicats différents, afficher des majorités pour les mêmes employés. L'expérience veut, dans les cas de maraudage syndical, qu'un scrutin soit ordonné afin que les employés puissent choisir librement dans l'abri de l'isoloir, qui ils veulent avoir comme représentant. Cependant, comme le point de mire est le désir des employés, il se peut que dans des situations exceptionnelles il ne soit pas nécessaire, comme ce fut le cas pour Les Moulins Maple Leaf Limitée, 23 di 114, d'ordonner un vote, le désir des employés ne laissant aucun doute. Comme nous l'avons également mentionné, nous pouvons exceptionnellement être appelé à décréter un vote en vertu de l'article 127(1) dans le cas où le requérant déplaçant n'a pas une majorité au moment du dépôt de sa requête en accréditation.»

Syndicat des travailleurs en Communication du Canada et Syndicat des communications du Canada et Bell Canada.

Dossier 530-437; décision du 30 avril 1979 (no 192); panel du Conseil: Me Claude H. Foisy, vice-président, MM. Lorne E. Shaffer et Jacques Archambault, membres. 


\section{Le droit à un procès juste et impartial pour un membre du syndicat}

Le Conseil a accueilli une plainte de pratique déloyale qui avai été logée contre le syndicat à l'effet qu'il avait appliqué envers le plaignant ses normes de discipline de manière discriminatoire en l'expulsant de ses rangs pour une période de dix ans.

Le Conseil a conclu que le plaignant n'avait pas eu le procès juste et impartial auquel il aurait eu droit en vertu des status du syndicat, certains membres accusateurs ayant participé à la prise de décision lors de la tenue de l'appel qu'il avait formulé devant l'assemblée annuelle des membres suite à sa suspension.

Constatant que l'article $185(\mathrm{~g})$ du Code avait été violé, le Conseil a ordonné la réintégration du plaignant.

Val Udvarhely et l'Association du personnel naviguant des lignes aériennes canadiennes.

Dossier 530-399; décision du 8 juin 1979 (no 200); panel du Conseil: Me Claude H. Foisy, vice-président, Me Robert J. Arseneau et $M$. Cleve Kidd, membres.

\section{LE CODE DU TRAVAIL DU QUÉBEC}

\section{5 ans après ...}

Introduction: Rodrigue BLOUIN, Jean-Paul DESCHENES, Jean SEXTON, Alain VINET - Le Code du travail du Québec: 15 ans après ..., Rodrigue BLOUIN - Le droit à l'accréditation: la majorité est-elle toujours absolue? Robert GAGNON -Commentaires, Roger THIBODEAU - La liberté syndicale: les droits collectifs et les droits individuels, Jean BERNIER - Commentaires, Louise MAILHOT, Colette MATTEAU - L'exercice du droit de grève et de lock-out est-il encore possible?, Pierre VERGE - Panel: Secteur public, secteur privé, Norbert RODRIGUE, Réjean LAROUCHE, Ghislain DUFOUR - Le processus de la négociation collective et l'arbitrage obligatoire d'une première convention collective, Jean-Paul DESCHENES - Commentaires, Marc LAPOINTE - Le système d'arbitrage de grief est-il désuet?, Jacques DUPONT - Commentaires, Michel DROLET, Robert PAQUET - Le Code de 1964, a-t-il été trahi dans son économie fondamentale?, Fernard MORIN, Marcel PEPIN, Gérard DION, Charles PERREAULT

$$
1 \text { volume, } 267 \text { pages - Prix }-\$ 15.00
$$

Les Presses de l'Université Laval

Cité universitaire

Québec, P.Q., Canada

G1K 7R4 